\title{
Limit on the Temporal Variation of the Fine-Structure Constant Using Atomic Dysprosium
}

\author{
A. Cingöz, ${ }^{1}$ A. Lapierre, ${ }^{1}$ A.-T. Nguyen, ${ }^{2}$ N. Leefer, ${ }^{1}$ D. Budker, ${ }^{1,3}$ S. K. Lamoreaux,, * and J. R. Torgerson ${ }^{2}$ \\ ${ }^{1}$ Department of Physics, University of California at Berkeley, Berkeley, California 94720-7300, USA \\ ${ }^{2}$ Los Alamos National Laboratory, Physics Division, \\ P-23, MS-H803, Los Alamos, New Mexico 87545, USA \\ ${ }^{3}$ Nuclear Science Division, Lawrence Berkeley National Laboratory, Berkeley, California 94720, USA
}

(Dated: July 20, 2018)

\begin{abstract}
Over a period of eight months, we have monitored transition frequencies between nearly degenerate, opposite-parity levels in two isotopes of atomic dysprosium (Dy). These transition frequencies are highly sensitive to temporal variation of the fine-structure constant $(\alpha)$ due to relativistic corrections of large and opposite sign for the opposite-parity levels. In this unique system, in contrast to atomic-clock comparisons, the difference of the electronic energies of the opposite-parity levels can be monitored directly utilizing a radio-frequency (rf) electric-dipole transition between them. Our measurements show that the frequency variation of the $3.1-\mathrm{MHz}$ transition in ${ }^{163} \mathrm{Dy}$ and the $235-\mathrm{MHz}$ transition in ${ }^{162} \mathrm{Dy}$ are $9.0 \pm 6.7 \mathrm{~Hz} / \mathrm{yr}$ and $-0.6 \pm 6.5 \mathrm{~Hz} / \mathrm{yr}$, respectively. These results provide a value for the rate of fractional variation of $\alpha$ of $(-2.7 \pm 2.6) \times 10^{-15} \mathrm{yr}^{-1}(1 \sigma)$ without any assumptions on the constancy of other fundamental constants, indicating absence of significant variation at the present level of sensitivity.
\end{abstract}

PACS numbers: 06.20.Jr, 32.30.Bv

Modern theories attempting to unify gravitation with the other fundamental interactions have renewed interest in experimental searches for temporal and spatial variation of fundamental constants. These theories allow for, or even predict, such variation, which would violate Einstein's Equivalence Principle [1]. Recently, evidence for variation of $\alpha$ over cosmological time scales was discovered in absorption spectra of light from quasars [2, 3]. The result in Ref. [3] corresponds to $\dot{\alpha} / \alpha=(6.40 \pm 1.35) \times$ $10^{-16} / \mathrm{yr}$ assuming a linear shift over $10^{10}$ years. However, more recent measurements present sensitive results that are consistent with no variation [4, [5]. On a geological time scale of $10^{9}$ years, a test for variation of $\alpha$ comes from analyses of fission products of a natural reactor in Oklo (Gabon). There is a discrepancy between earlier analyses [6, 7], which are consistent with no variation, and the analysis in Ref. 8], where a variation was reported. However, a recent full Monte Carlo simulation of the reactor [9] reports a result consistent with no variation at the level of $1.2 \times 10^{-17} / \mathrm{yr}$.

Observational measurements are difficult to interpret due to numerous assumptions and uncontrollable systematic uncertainties. Laboratory searches (see, for example, Refs. 10, 11, 12, 13]), which probe variations on the time scale of years, are easier to interpret since they are repeatable, and systematic uncertainties can be studied by changing experimental conditions. The best laboratory limit of $|\dot{\alpha} / \alpha|<1.2 \times 10^{-15} /$ yr was obtained from a comparison of a $\mathrm{Hg}^{+}$optical clock to a Cs frequency standard [10]. This result assumes that other constants, in particular $m_{e} / m_{p}$, do not vary in time [14]. Interestingly, evidence for variation of this parameter on astronomical time scale was recently reported in Ref. [15].
The best limit that is independent of assumptions regarding other constants was obtained by combining this result with a comparison of $\mathrm{Yb}^{+}$optical clock and a Cs frequency standard [13]. This measurement gives $|\dot{\alpha} / \alpha|<2.2 \times 10^{-15} /$ yr.

In this Letter, we report a limit on the temporal variation of $\alpha$ that is independent of other constants. This result is based on observing, over a period of eight months, rf-transitions between nearly degenerate, opposite-parity levels in atomic dysprosium, as suggested in Ref. [16].

The energy of an atomic level can be expressed as

$$
E=E_{0}+q\left(\alpha^{2} / \alpha_{0}^{2}-1\right),
$$

where $E_{0}$ is the present-day energy, $\alpha_{0}$ is the presentday value of the fine-structure constant, and $q$ is a level dependent coefficient that determines the sensitivity to variations in $\alpha$ [17]. A theoretical calculation has found that the $q$-coefficients for the two nearly degenerate, opposite-parity levels in Dy (Fig. 1, levels $A$ and $B$ ) are large and of opposite sign [17]. For the even-parity level (level $A), q_{A} / h c \simeq 6 \times 10^{3} \mathrm{~cm}^{-1}$, while for the oddparity level (level $B), q_{B} / h c \simeq-24 \times 10^{3} \mathrm{~cm}^{-1}$. Since explicit uncertainties are not quoted for these numbers, they are assumed to be exact in the determination of the result [18].

Another parametrization of frequency and sensitivity factor is often used for atomic-clock comparisons, in which an electronic transition frequency is expressed as

$$
\nu=R_{\infty} A F(\alpha),
$$

where $R_{\infty}$ is the Rydberg constant expressed in units of frequency, $A$ is a dimensionless atomic structure factor independent of $\alpha$, and $F(\alpha)$ is a factor that includes all 


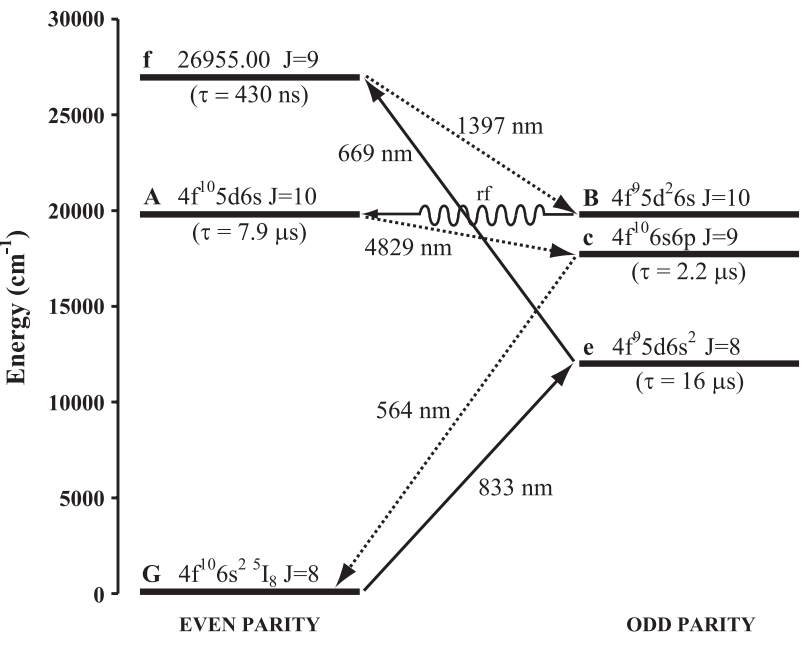

FIG. 1: Relevant levels and transitions in atomic dysprosium used to populate level $B$ and detect the population of level $A$. Solid lines indicate laser excitation; dashed lines indicate spontaneous emission; wavy line indicates rf electric field.

relativistic as well as many-body effects which depend on $\alpha$ [19]. The change in a transition frequency can be related to a fractional change in $\alpha$ as

$$
\frac{\dot{\nu}}{\nu}=\frac{d}{d t} \ln \nu=\left[\alpha \frac{\partial \ln F}{\partial \alpha}\right] \frac{\dot{\alpha}}{\alpha} .
$$

The expression $\alpha \partial \ln F / \partial \alpha$ is the sensitivity of a transition to variation in $\alpha$. Using this parametrization, the q-coefficients for atomic Dy can be recast (for $\alpha \approx \alpha_{0}$ ) to give $\alpha \partial \ln F / \partial \alpha=2 q /(h \nu)$, where $h \nu$ is the energy of level $A$ or $B$ with respect to the ground state. This gives a sensitivity of 0.6 and -2.4 for level $A$ and $B$, respectively. These values are comparable, for example, to the sensitivity of -3.2 for the transition used in the $\mathrm{Hg}^{+}$ optical clock [20].

In atomic-clock comparisons, the observable quantity is the ratio of the two frequencies being compared. A unique aspect of the Dy system is that the direct observable quantity is the difference $\nu_{B}-\nu_{A}$, due to the fact that an electric-dipole transition can be induced between the two levels using a rf electric field. The time variation of the transition frequency between levels $A$ and $B$ is

$$
\dot{\Delta \nu}=\dot{\nu}_{B}-\dot{\nu}_{A}=2 \frac{q_{B}-q_{A}}{h} \frac{\dot{\alpha}}{\alpha} \sim-1.8 \times 10^{15} \mathrm{~Hz} \frac{\dot{\alpha}}{\alpha} .
$$

For instance, $|\dot{\alpha} / \alpha|=10^{-15} /$ yr implies $|\dot{\Delta} \nu| \simeq 2 \mathrm{~Hz} / \mathrm{yr}$.

There are several advantages to using the nearly degenerate levels in Dy. Because most of the transition frequencies are $\sim 1 \mathrm{GHz}$ or smaller, direct frequency counting techniques can be used. This allows for the comparison of two electronic transitions without the need for optical frequency combs or transfer cavities. A preliminary analysis of statistical and systematic uncertainties shows that the measurement of the transition frequency and the control of the systematics at a $\mathrm{mHz}$ level is feasible, which corresponds to an ultimate sensitivity of $|\dot{\alpha} / \alpha| \sim 10^{-18} /$ yr for two measurements separated by a year's time 21]. A mHz resolution on a transition frequency of $1 \mathrm{GHz}$ requires a relatively modest fractional stability of $10^{-12}$ for the reference frequency standard. This also means that the results are insensitive to variation of the Cs reference frequency due to changes in the values of fundamental constants since the experimental upper limit on this relative variation rate is $\sim 10^{-15} \mathrm{yr}^{-1}$ (see, for example, [1] $)$. In addition, because the isotope shifts and hyperfine splittings are on the order of the transition frequencies, it is possible to work with multiple rf transitions corresponding to the same electronic transition, including, in particular, transitions with energy differences of opposite sign. Since the variation in $\alpha$ only depends upon electronic energy levels [22], the variation of these $\mathrm{rf}$ transitions should have equal magnitude but opposite sign. This correlation can be used to detect and eliminate certain systematics. Currently, we monitor two such transitions: the $3.1-\mathrm{MHz}(F=10.5 \rightarrow F=10.5)$ transition in ${ }^{163} \mathrm{Dy}$ and the $235 \mathrm{-MHz}(J=10 \rightarrow J=10)$ transition in ${ }^{162}$ Dy.

The population and detection scheme is shown in Fig. 1. The long-lived $\left(\tau_{B}>200 \mu \mathrm{s}\right.$ [23]) level $B$ is populated by three transitions. The first two transitions are driven with 833- and 669-nm laser light, while the third transition involves spontaneous decay. Atoms are transferred to level $A$ with rf electric field referenced to a Cs frequency standard. Level $A$ decays to the ground state in two steps. Fluorescent light at $564 \mathrm{~nm}$ from the second decay is used for detection.

The atomic beam is described in Ref. [24]. It is produced by an effusive oven operating at $\sim 1500 \mathrm{~K}$. In addition to a multislit nozzle-array attached to the oven, two external collimators are used to collimate both the atomic beam and the oven light (in order to minimize the background due to scattered light from the oven). The resultant atomic beam has a mean velocity of $500 \mathrm{~m} / \mathrm{s}$ and a full-angle divergence of $\sim 0.2 \mathrm{rad}\left(1 / e^{2}\right.$-level).

The laser light at 833 and $669 \mathrm{~nm}$ is produced by a Ti:Sapphire ring laser and a ring dye laser with DCM dye, respectively. Since narrow-band continuous-wave lasers are inefficient at exciting atomic beams with weak collimation, an adiabatic passage technique is utilized to transfer the population to level $f$ (a description of this technique, as well as references to earlier work, are given in Ref. [25]). Briefly, the beams are diverged with cylindrical lenses to match the atomic-beam divergence. Due to the Doppler effect, the atoms experience a frequency chirp in laser detuning which transfers the population to the excited state with high efficiency. The laser-light and rf-interaction region is enclosed by a magnetic shield. The residual field in the interaction region is $\sim 1 \mathrm{mG}$.

The rf-generation and detection system is discussed in Ref. [26]. The frequency modulated rf field is generated by a synthesizer referenced to a commercial Cs frequency 
standard which is compared to a second clock (Rb oscillator locked to a digital cellular network signal) to monitor its stability. The short term fractional stability of the clocks is $\sim 10^{-11}$, and it approaches $10^{-12}$ in 10 minutes of integration. The modulation frequency is $10 \mathrm{kHz}$ with a modulation index of 1 . Since the rf-transition linewidth is $\sim 20 \mathrm{kHz}$, this modulation provides a fast sweep across the line shape, minimizing the effect of fluctuations such as those due to laser power drifts or density fluctuations of the atomic beam. The modulation is provided by the reference output of a lock-in amplifier which demodulates the signal from a photomultiplier tube used to detect the fluorescence. The line shape of the first- and secondharmonic outputs of the lock-in amplifier is, respectively, an odd function with zero crossing on resonance and an even function with a maximum on resonance. In order to reduce drifts further, the ratio of these two is used to measure the transition frequency, which is extracted by a two-step process described in detail in Ref. 26].

There are several imperfections that affect the stability of our transition-frequency measurements. The largest effect is due to the combination of the residual magnetic field with the laser-light polarization imperfections. During normal operation, the polarizations of both laser beams are set to linear, which should lead to symmetrically populated magnetic sublevels. However, residual light ellipticity leads to atomic orientation, which, in the presence of the residual magnetic field, leads to asymmetric broadening of the line and causes apparent shifts 21]. In systematic studies where we deliberately used circularly polarized light, shifts in the transition frequencies as large as $\pm 160 \mathrm{~Hz}$ were observed. The linear polarizations of the laser beams are determined by calcite polarizers with $10^{-5}$ extinction ratio outside the vacuum chamber. However, the beams must travel through optical windows and lenses utilized for adiabatic passage population technique (see above) before reaching the interaction region. A small amount of stress-induced birefringence on these lenses was discovered by deliberate misalignment of the laser beams. The amount of ellipticity depended upon where the beam sampled a lens. The residual systematic uncertainties due to this effect range from 2 to $5 \mathrm{~Hz}$ on different experimental runs with the smaller uncertainty corresponding to later runs in which the lens mounts were modified to relieve the stress.

In addition to the residual magnetic field, the magnetic shielding was inadvertently magnetized in the April 2006 runs during studies utilizing external magnetic-field coils. Since the amount of magnetization and the resultant shifts in the transition frequencies were unknown at the time, systematic uncertainties have been assigned by driving the magnetization to saturation and noting the maximum shift induced for both field polarities. The uncertainty due to this effect is $7.5 \mathrm{~Hz}$. The shields were demagnetized at the beginning of each subsequent run.

Another important systematic uncertainty is due to the collisions of Dy atoms with the background gas in the vacuum chamber. Collisional shift rates for the rf transitions due to various gases were determined in Ref. 26]. The pressure in the vacuum chamber is $\sim 5 \times 10^{-7}$ Torr when the Dy oven is off. When the oven is turned on, the pressure rises to $\sim 2 \times 10^{-6}$ Torr, limited by $\mathrm{H}_{2}$ outgassing from the oven. In Ref. 26], the observed shift rates for $\mathrm{H}_{2}$ were found to be consistent with zero. However, the pressure of other gases, such as $\mathrm{N}_{2}$, also increases at the level of $\sim 4 \times 10^{-7}$ Torr when the oven is turned on, and continually decreases and stabilizes after $\sim 6$ hours of continuous oven operation. The partial pressures are monitored with a residual gas analyzer (RGA), and the transition frequencies are corrected for the presence of $\mathrm{N}_{2}, \mathrm{O}_{2}, \mathrm{H}_{2}$, and Ar using the shift rates measured for each gas. The variation in the pressure of other gases such as $\mathrm{H}_{2} \mathrm{O}$ and $\mathrm{CO}_{2}$ are $\sim 10^{-7}$ Torr or smaller, and no significant correlation between these pressure variations and the transition frequency is observed. The total correction is $<2 \mathrm{~Hz}$ at any time during each run. The results also include small uncertainties $(<0.6 \mathrm{~Hz})$ due to oven temperature variations, which may indicate a possible effect of Dy intra-beam collisions [26].

Finally, there are systematic effects associated with $\mathrm{rf}$ electric-field inhomogeneities. The rf electrodes are fed by twisted-pair wires at one corner. Numerical simulations show that with this feed, at rf wavelengths comparable to the size of the electrodes $(\sim 10 \mathrm{~cm})$, spatially varying amplitude and phase inhomogeneities become substantial. These inhomogeneities can lead to drifts in the transition frequency when they are combined with changes in the atomic beam velocity distribution. This effect was studied experimentally by deliberate detuning of the laser frequencies from the optical resonances. This leads to changes in the transverse velocity distribution of the excited atoms due to imperfect adiabatic passage. For the two transitions considered in this study, shifts of $<0.5 \mathrm{~Hz}$ for $1 \mathrm{MHz}$ detuning in the laser frequencies were observed. To keep this effect at the $1 \mathrm{~Hz}$ level during the runs, the laser frequencies were periodically retuned ( $\sim$ every 2 minutes) to the center of their respective transitions. To check for slow variations in the beam velocity distribution due to other changes such as collimator clogging, this study was repeated several times over a two-year period, during which the oven was reloaded and collimators cleaned several times. No significant change in the size or dependence of the effect was observed.

All other systematics considered in Ref. 21] were estimated to be smaller than $1 \mathrm{~Hz}$ for the specific conditions of the current experiment. Many of these systematics, such as AC Stark shifts and oven black-body radiation shifts, were studied experimentally by exaggerating certain imperfections and were found to be negligible.

We have measured the two rf transition frequencies in the course of seven runs over eight months. Fig- 

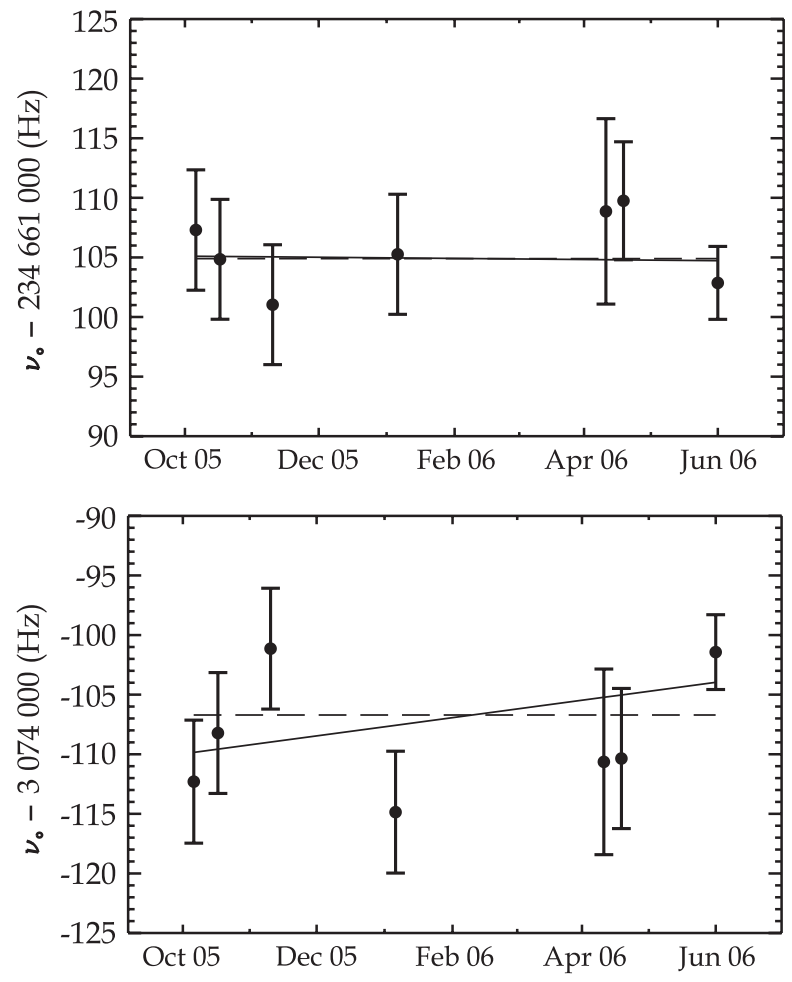

FIG. 2: Measured transition frequencies for the $235-\mathrm{MHz}$ and 3.1-MHz transitions over an eight-month period. The data have been corrected for collisional shifts. The solid lines are the least-squares linear fit to the data. The dashed lines are the least-squares fit to a constant function.

ure 2 shows the results of these measurements corrected for collisional shifts. Uncertainties are due both to statistical and aforementioned systematic uncertainties. Least-squares linear fit to the data points gives slopes of $-0.6 \pm 6.5 \mathrm{~Hz} / \mathrm{yr}$ and $9.0 \pm 6.7 \mathrm{~Hz} / \mathrm{yr}$ for the 235 $\mathrm{MHz}$ and $3.1-\mathrm{MHz}$ transitions, respectively. According to Eq. (4), these results correspond to $\dot{\alpha} / \alpha=$ $(-0.3 \pm 3.6) \times 10^{-15} \mathrm{yr}^{-1}$ for the $235-\mathrm{MHz}$ transition and $(-5.0 \pm 3.7) \times 10^{-15} \mathrm{yr}^{-1}$ for the $3.1-\mathrm{MHz}$ transition. As mentioned earlier, because the energy differences for these two transitions are of opposite sign, these two data sets can be combined to construct sum and difference frequency plots. The sum frequency slope, which should be insensitive to a variation in $\alpha$, is $8.1 \pm 9.4 \mathrm{~Hz} / \mathrm{yr}$ and consistent with zero. The difference frequency, which is twice as sensitive to a variation in $\alpha$ as the individual frequencies, gives the final result of $\dot{\alpha} / \alpha=(-2.7 \pm 2.6) \times 10^{-15} \mathrm{yr}^{-1}$, consistent $(1 \sigma)$ with no variation at the present level of sensitivity.

In conclusion, we have presented the first result of a direct measurement of the temporal variation of $\alpha$ with atomic dysprosium. This result is of comparable uncertainty to the present best laboratory result that is independent of other fundamental constants. However, in our case the interpretation does not require compari- son with different measurements to eliminate dependence on other constants, and provides an alternative to measurements that utilize state-of-the-art atomic optical frequency clocks. The present uncertainty is dominated by systematic effects primarily due to polarization imperfections coupled to the residual magnetic field, collisional shifts, and rf electric-field inhomogeneities. A significant improvement is expected from a new apparatus under construction which will provide better control over these, as well as other, systematic effects expected to be important to achieve better than $1-\mathrm{Hz}$ sensitivity. Ultimately, mHz-level sensitivity may be achievable with this method [21].

We thank V. V. Yashchuk, D. F. Jackson Kimball, and D. English for valuable discussions. This work was supported in part by the University of California - Los Alamos National Laboratory CLC program, NIST Precision Measurement Grant, Los Alamos National Laboratory LDRD, and by grant RFP1-06-15 from the Foundational Questions Institute (fqxi.org).

* Present address: Department of Physics, Yale University, New Haven, Connecticut 06520-8120, USA

[1] J.-P. Uzan, Rev. Mod. Phys. 75, 403 (2003).

[2] J. K. Webb et al., Phys. Rev. Lett. 87, 091301 (2001).

[3] M. T. Murphy et al., Mon. Not. R. Astron. Soc. 345, 609 (2003).

[4] R. Quast et al., Astron. Astrophys. 415, L7 (2004).

[5] R. Srianand, H. Chand, P. Petitjean, and B. Aracil, Phys. Rev. Lett. 92, 121302 (2004).

[6] T. Damour and F. Dyson, Nucl. Phys. B480, 37 (1996).

[7] Y. Fujii et al., Nucl. Phys. B573, 377 (2000).

[8] S. K. Lamoreaux and J. R. Torgerson, Phys. Rev. D 69, 121701(R) (2004).

[9] C. R. Gould, E. I. Sharapov, and S. K. Lamoreaux, Phys. Rev. C 74, 024607 (2006).

[10] S. Bize et al., Phys. Rev. Lett. 90, 150802 (2003).

[11] H. Marion et al., Phys. Rev. Lett. 90, 150801 (2003).

[12] M. Fischer et al., Phys. Rev. Lett. 92, 230802 (2004).

[13] E. Peik et al., Phys. Rev. Lett. 93, 170801 (2004).

[14] In fact, recent theories predict that the fractional variation in $m_{e} / m_{p}$ is $\sim 40$ times larger than the fractional variation in $\alpha$ [X. Calmet and H. Fritzsch, Eur. Phys. J. C 24, 639 (2002)].

[15] E. Reinhold et al., Phys. Rev. Lett. 96, 151101 (2006).

[16] V. A. Dzuba, V. V. Flambaum, and J. K. Webb, Phys. Rev. Lett. 82, 888 (1999).

[17] V. A. Dzuba, V. V. Flambaum, and M. V. Marchenko, Phys. Rev. A 68, 022506 (2003).

[18] The uncertainty of these numbers is expected to be $\sim 20 \%$ (private communication with V. V. Flambaum).

[19] J. D. Prestage, R. L. Tjoelker, and L. Maleki, Phys. Rev. Lett. 74, 3511 (1995).

[20] V. A. Dzuba, V. V. Flambaum, and J. K. Webb, Phys. Rev. A 59, 230 (1999).

[21] A. T. Nguyen, D. Budker, S. K. Lamoreaux, and J. R. Torgerson, Phys. Rev. A 69, 22105 (2004).

[22] There are differences due to the hyperfine interaction and 
isotope shifts, but these are several orders of magnitude smaller.

[23] D. Budker, D. DeMille, E. D. Commins, and M. S. Zolotorev, Phys. Rev. A 50, 132 (1994).

[24] A. T. Nguyen, D. Budker, D. DeMille, and M. Zolotorev, Phys. Rev. A 56, 3453 (1997).
[25] A. T. Nguyen, G. D. Chern, D. Budker, and M. Zolotorev, Phys. Rev. A 63, 013406 (2000).

[26] A. Cingöz, A.-T. Nguyen, D. Budker, S. K. Lamoreaux, A. Lapierre, and J. R. Torgerson, Phys. Rev. A 72, 063409 (2005). 